\title{
Percepções de acadêmicas de enfermagem acerca da assistência ao paciente cirúrgico com sepse na clínica cirúrgica
}

\author{
Perceptions of nursing academics about surgical patient assistance with sepsis in the surgical clinic \\ Percepciones de los académicos de enfermería sobre la asistencia al paciente quirúrgico con sepsis \\ en la clínica quirúrgica
}

Luzia Gabriela Marques Costa

ORCID: https://orcid.org/0000-0001-7025-1970

Centro COVID-19 de Rio de Contas, Brasil

E-mail: gabrielamarquescosta92@ gmail.com

Mylena Lima Malheiros

ORCID: https://orcid.org/0000-0001-8995-1993

Universidade do estado da Bahia, Brasil

E-mail: mylalima0802@gmail.com

Darlyane Antunes Macedo

ORCID: https://orcid.org/0000-0001-9342-3536

Universidade do Estado da Bahia, Brasil

E-mail: damacedo@uneb.br

Ivanete Fernandes do Prado

ORCID: https://orcid.org/0000-0001-9188-4275

Universidade do Estado da Bahia, Brasil

E-mail: iprado@uneb.br

Ricardo Bruno Santos Ferreira

ORCID: https://orcid.org/0000-0003-0614-4817

Universidade do Estado da Bahia, Brasil

Hospital do Rim de Guanambi, Brasil.

E-mail: ricardobrunoenf@gmail.com

\begin{abstract}
Resumo
Objetivo: o presente estudo tem como objetivo descrever as percepções de acadêmicas de enfermagem acerca da assistência ao paciente com sepse em uma clínica cirúrgica. Metodologia: Trata-se de um relato de experiência, desenvolvido a partir da assistência ao paciente com sepse durante as práticas curriculares no estágio supervisionado do Curso de Enfermagem em um Hospital Geral do Estado da Bahia. Os dados foram coletados no período de 21 de março a 28 de abril de 2019, a partir das situações observadas durante a assistência ao paciente com sepse, diálogo entre os profissionais, pacientes e familiares. Os registros das informações foram realizados em diário de campo. Resultados e discussão: Percebeu-se que a assistência ao paciente deve ser realizada em unidades de terapia intensiva, uma vez que o tratamento exige cuidado especializado, com utilização de tecnologias e avaliação contínua. Em municípios ou unidades hospitalares sem essa estrutura especializada é necessário que o núcleo de educação permanente local realize capacitação periódica e institucionalize protocolos de atendimento a fim de padronizar a assistência prestada, com foco no diagnóstico precoce. Dada a complexidade do atendimento, a assistência de enfermagem é fundamental em todas as fases de atendimento, desde o diagnóstico e tratamento, até a comunicação e construção de vínculo com familiares. Conclusão: é fundamental criar estratégias para capacitação da equipe multidisciplinar e protocolos visando o diagnóstico precoce e tratamento ao paciente com sepse fora da unidade de terapia intensiva.
\end{abstract}

Palavras-chave: Percepção; Sepse; Assistência à saúde; Cuidados de enfermagem.

\begin{abstract}
Objective: the present study aims to describe the perceptions of nursing students about assistance to patients with sepsis in a surgical clinic. Methodology: This is an experience report, developed from the assistance to patients with sepsis during curricular practices in the supervised internship of the Nursing course at a general hospital in the state of Bahia. Data were collected from March 21 to April 28, 2019, from the situations observed during care for patients with sepsis, dialogue between professionals, patients and family members. The information was recorded in a field diary. Results and discussion: It was realized that patient care should be carried out in intensive care units, since treatment requires specialized care, with the use of technologies and continuous assessment. In municipalities or hospital units without this specialized structure, it is necessary for the local permanent education nucleus to carry out periodic training and institutionalize care protocols in order to standardize the care provided, focusing on early
\end{abstract}


diagnosis. Given the complexity of care, nursing care is essential in all phases of care, from diagnosis and treatment, to communication and building bonds with family members. Conclusion: it is essential to create strategies for training the multidisciplinary team and protocols aimed at early diagnosis and treatment of patients with sepsis outside the intensive care unit.

Keywords: Sepsis; Perceptión; Delivery of health care; Nursing care.

\section{Resumen}

Objetivo: el presente estudio tiene como objetivo describir las percepciones de estudiantes de enfermería sobre la atención a pacientes con sepsis en una clínica quirúrgica. Metodología: Se trata de un relato de experiencia, desarrollado a partir de la asistencia a pacientes con sepsis durante las prácticas curriculares en el internado supervisado del curso de Enfermería en un hospital general del estado de Bahía. Se recolectaron datos del 21 de marzo al 28 de abril de 2019, a partir de las situaciones observadas durante la atención a pacientes con sepsis, diálogo entre profesionales, pacientes y familiares. La información se registró en un diario de campo. Resultados y discusión: Se reconoció que la atención al paciente debe realizarse en unidades de cuidados intensivos, ya que el tratamiento requiere cuidados especializados, con el uso de tecnologías y evaluación continua. En los municipios o unidades hospitalarias sin esta estructura especializada, es necesario que el núcleo de educación permanente local realice capacitaciones periódicas e institucionalice los protocolos de atención con el fin de estandarizar la atención brindada, con foco en el diagnóstico temprano. Dada la complejidad del cuidado, el cuidado de enfermería es fundamental en todas las fases del cuidado, desde el diagnóstico y el tratamiento, hasta la comunicación y la construcción de vínculos con los familiares. Conclusión: es fundamental la creación de estrategias de formación del equipo multidisciplinario y protocolos dirigidos al diagnóstico y tratamiento precoz de los pacientes con sepsis fuera de la unidad de cuidados intensivos.

Palablas claves: Sepsis; Percepción; Prestación de atención de salud; Atención de enfermería.

\section{Introdução}

A sepse é definida como um conjunto de manifestações clínicas graves, gerados por uma resposta exacerbada do organismo contra uma infecção. Trata-se de um grave problema de saúde global, possuindo elevadas taxas de morbimortalidade (Fernandes et al, 2018; Silva \& Souza, 2018).

Trata-se de uma condição clínica grave, capaz de desencadear disfunções cardiovasculares, respiratória, neurológica, renal, hematológica, gastrointestinal e endócrina. O choque séptico, caracterizado como falência circulatória aguda, é a principal complicação do quadro séptico, e, uma vez instalado aumenta significativamente o risco de morte (Viana; Machado \& Souza, 2020).

Segundo o Instituto Latino Americano de Sepse (ILAS) estima-se uma incidência anual de, aproximadamente, 15 a 17 milhões de novos casos de sepse no mundo, dentre as quais, 5 milhões evoluem para óbito. Destaca-se que, dentre as pessoas que evoluíram para óbito,42,2\% morreram nas unidades de pronto socorro de instituições públicas, o que reflete a dificuldade, tanto no acesso às unidades de terapia intensiva, quanto no diagnóstico precoce (Viana; Machado \& Souza, 2020).

Nesse sentido, é fundamental a organização dos serviços de saúde e capacitação dos profissionais, com vistas a garantir um atendimento sistematizado, com manejo clínico individualizado. Acredita-se que, nesse contexto, a atuação coordenada e qualificada da equipe multidisciplinar é fundamental para acompanhamento da evolução do paciente e redução dos percentuais de morbimortalidade (Rosa et al, 2018).

Dessa forma, a consolidação de uma assistência qualificada perpassa diretamente pela reflexão e discussão do contexto da assistência atual nas unidades de saúde. Essa reflexão está diretamente conectada ao processo de ação-reflexãoação, essencial para construção da prática do cuidado em enfermagem (Waldow, 2009).

Considerando o exposto, o presente estudo tem como objetivo descrever as percepções das acadêmicas de enfermagem sobre a assistência ao paciente com sepse em uma clínica cirúrgica. 


\section{Metodologia}

O estudo apresenta uma abordagem qualitativa, descritiva e reflexiva, do tipo relato de experiência. Segundo Daltro e Faria (2019) trata-se de uma modalidade qualitativa de produção do conhecimento, que valoriza e destaca a explicação descritiva, interpretativa e compreensiva de um determinado dado. A escolha da abordagem qualitativa foi fundamental para atender ao objetivo, uma vez que se trata de um estudo construído a partir da experiência, descrição e interpretação dos autores de uma determinada realidade (Pereira et al; 2018).

A vivência do fenômeno em questão ocorreu durante as atividades práticas da disciplina Estágio Supervisionado II, componente do $10^{\circ}$ semestre do curso de bacharelado em enfermagem da Universidade do Estado da Bahia (UNEB), Campus XII.

O cenário foi um hospital geral público, de porte médio, localizado na região sudoeste da Bahia. A instituição conta com 140 leitos, sendo considerado referência de atendimento às urgências e emergências para 31 municípios. Além disso, conta com um ambulatório especializado, recursos humanos e tecnológicos para a prestação de assistência nas clínicas: médica, cirúrgica, pediátrica, obstétrica, UTI neonatal, UTI Adulto e UCI Neonatal.

O estudo foi construído durante as práticas na Clínica Cirúrgica, no período de 21 de março a 8 abril de 2019. Os dados foram coletados a partir das situações observadas durante a assistência ao paciente com sepse, diálogo entre os profissionais, pacientes e familiares. Os registros das informações foram realizados em diário de campo, no qual anotou-se, diariamente, as informações relacionadas ao cuidado ao paciente séptico.

Essas informações foram guiadas pelas atividades assistenciais e gerenciais desenvolvidas durante o estágio, o que possibilitou a realização, observação e avaliação das atividades que implicam no cuidado integral de enfermagem ao paciente.

Por tratar-se de um relato de experiência, sem identificação de sujeitos, instituições ou intervenções diretas, não houve necessidade de submissão no comitê de ética e pesquisa.

\section{Resultados e Discussão}

A Clínica Cirúrgica, local do estudo, possui 9 enfermarias e 4 isolamentos, totalizando 34 leitos para internação de pacientes adultos e crianças acima de dois anos. Inicialmente, notou-se que os leitos de enfermaria não se mostraram adequados para o tratamento de pessoas com sepse, uma vez que se trata de uma condição clínica grave, que exige cuidado especializado, avaliação contínua e disponibilidade de tecnologias específicas.

A necessidade de tratamento em Unidades de Terapia Intensiva (UTI) se justifica pelas altas taxas de mortalidade por sepse, cerca de 30\% em 2016. Em situações em que há evolução para choque séptico, a mortalidade pode alcançar até 65,3\% (Lobo et al; 2019).

Além da inadequada estrutura nas enfermarias para atendimento ao paciente séptico, verificou-se que o enfermeiro, além de ser responsável pela supervisão dos técnicos de enfermagem, possui grande demanda de procedimentos privativos na Clínica Cirúrgica, o que limita a assistência ao paciente com sepse no setor.

Tal afirmativa também foi evidenciada nos estudos de Santana, Marques e Spolidoro (2017), Maissiat et al (2015), Silva et al (2017) e Lobo et al (2019). Segundo os autores, pacientes que dependem de cuidado integral, especializado e individualizado devem ser realizados em Unidade de Terapia Intensiva, devido à limitação de equipamentos, tecnologias e profissionais em outros setores.

O paciente assistido era pós cirúrgico de laparotomia para investigar dor em região abdominal e evoluiu com quadro de febre, calafrios, astenia, falta de apetite, dor em região abdominal, deiscência de pontos em ferida operatória, secreção seropurulenta em incisão e odor fétido, sendo constatado o quadro de sepse pelo médico responsável. 
Durante o acompanhamento ao paciente foi possível observar, acompanhar e realizar os procedimentos privativos do (a) enfermeiro (a) como passagem de sonda vesical, realização de curativos, monitoramento dos sinais vitais, administração de antibióticos, bem como o acompanhamento direto aos pacientes e a evolução do quadro clínico junto à equipe multiprofissional.

A detecção precoce e tratamento da sepse deve ser feita nas primeiras seis horas de atendimento devido à alta taxa de mortalidade (Silva \& Souza, 2018). Por isso, é fundamental que as instituições de saúde elaborem e institucionalizem, tanto um protocolo específico para rastreamento dos pacientes com potencial séptico, quanto para o manejo clínico dos pacientes diagnosticados, algo que não foi evidenciado na Clínica cirúrgica.

Há necessidade, por exemplo, de institucionalizar medidas de assistência focada triagem para pacientes suspeitos e implantação dos pacotes de atendimento de três horas para pacientes com sepse ou choque séptico, como preconiza o Ilas (2019) através da coleta de lactato, hemoculturas, início de antibioticoterapia de amplo espectro e reposição volêmica inicialmente, e pacote de seis horas, com utilização de vasopressor, com reavaliação da volemia, perfusão tecidual e níveis de lactato em pacientes com hipotensão persistente.

É necessário ponderar que, apesar da recomendação de tratamento de pacientes graves na UTI, a realidade brasileira não consegue atender à todas as necessidades devido a superlotação das unidades hospitalares, o que reflete, rotineiramente, na assistência à pacientes graves nas enfermarias e emergências.

Por isso, Westphal e Lino (2015) sugerem a criação de equipes de resposta rápida ou extensão da atuação extramuros da UTI como estratégia para assistência especializada à pacientes graves, o que facilitaria a assistência especializada. Contudo, tal sugestão pode enfrentar resistência nas instituições, dado o limitado número de profissionais. Desse modo, acredita-se que os núcleos de educação permanente e/ou coordenações dos hospitais devem criar espaços de diálogo entre os diversos setores afim de institucionalizar protocolos para identificação e tratamento precoce dos pacientes com sepse.

Há evidências de que a criação de ferramentas de triagem em enfermarias aumente substancialmente a identificação de casos de sepse e contribua para reduzir a mortalidade, o que reforça os achados supracitados (Branco et al, 2020).

No que se refere às ações de gerenciamento do cuidado, apesar de não ser institucionalizado o processo de enfermagem, foi realizado exame físico e evolução diária, avaliação da ferida, prescrição e realização de curativos durante todo o período do estágio.

Segundo Veras et al (2019) as ações de enfermagem são indispensáveis no gerenciamento do protocolo clínico de sepse, pois é a equipe mais próxima do paciente na assistência, desde sua admissão na unidade hospitalar até a alta, o que garante maior possibilidade em detectar precocemente os sinais e sintomas da sepse.

Segundo resolução do Conselho Federal de Enfermagem (COFEN) n 358 o processo de enfermagem baseia-se em cinco etapas: coleta de dados, diagnóstico de enfermagem, planejamento de enfermagem, implementação e avaliação. Como evidenciado nos estudos de Porto, Leal e Ferreira (2018) o processo de enfermagem auxilia na abordagem holística e individualizada, além de conferir cientificidade à assistência de enfermagem. Ademais, há evidências importantes da assistência de enfermagem sistematizada ao paciente com sepse (Branco et al, 2020)

Além das ações assistenciais mencionadas, cabe ao profissional de enfermagem a administração de antibióticos e coleta de hemoculturas da forma correta, o que exige capacitação profissional e grande articulação com a equipe médica. Segundo Rosa et al (2018) através da atuação da equipe de enfermagem é possível traçar diagnósticos e implantar medidas para o tratamento e prevenção de novos agravos.

Além disso, a comunicação é uma peça fundamental para a atenção holística uma vez que a sepse possui grande impacto psicológico nos pacientes e familiares, por se tratar de um diagnóstico com os parâmetros incertos de recuperação. 
Observou-se que esse aspecto não favorece o fortalecimento de vínculo entre a equipe de saúde e pacientes/familiares, os quais se julgam incapazes de lidar com as limitações impostas pelo ambiente hospitalar (Oliveira; Silva \& Silva, 2018).

Por isso, foi realizado diariamente abordagem aos familiares para explicar detalhadamente os cuidados prestados e escutar atentamente as aflições e questionamentos, o que facilitou a construção de vínculo durante o período do estágio.

Assim como em outros estudos, constatou-se que a construção do vínculo foi um importante instrumento auxiliador nas práticas do cuidado, uma vez que as informações obtidas através do paciente e dos familiares auxiliam para compreensão de suas vulnerabilidades, comportamentos de risco e contexto socioeconômico que posteriormente iram subsidiar e nortear o plano de cuidado e orientações no processo de recuperação, principalmente no ambiente domiciliar (Oliveira; Silva \& Silva, 2018).

Nota-se assim que a assistência de enfermagem é fundamental na assistência ao paciente com sepse. Contudo, tais ações devem ser realizadas de maneira articulada com a equipe interdisciplinar uma vez que se trata de um quadro complexo e desafiador.

\section{Conclusão}

Através do estudo foi possível refletir acerca da assistência ao paciente com sepse em uma unidade de clínica cirúrgica. Percebeu-se que a assistência ao paciente deve ser realizada em UTIs, uma vez que o tratamento exige cuidado especializado, com utilização de tecnologias e avaliação contínua.

Em municípios ou unidades hospitalares sem presença de UTI é necessário que o núcleo de educação permanente local realize capacitação periódica e institucionalize protocolos de atendimento a fim de padronizar a assistência prestada, com foco no diagnóstico precoce.

Dada a complexidade do atendimento, a assistência de enfermagem é fundamental em todas as fases de atendimento, desde o diagnóstico e tratamento, até a comunicação e construção de vínculo com familiares.

O fato de se tratar de um relato de experiência foi o principal limitante do estudo, não sendo possível a entrevista de profissionais e familiares acerca da assistência. Entretanto, diante do limitado número de estudos relacionados ao paciente com sepse, o estudo traz resultados que apontam, de maneira importante, para a direção de uma melhor assistência ao paciente com sepse fora da UTI. Ademais, o presente estudo sinaliza para a necessidade de construção de novas pesquisas voltadas para a construção e validação de ferramentas de sistematização da assistência à pessoas com sepse fora da UTI.

\section{Referências}

Branco, M. J. C. Lucas, A. P. M. Marques, R. M. D. \& Sousa, P. P. (2020). O papel do enfermeiro perante o paciente crítico com sepse. Revista Brasileira de Enfermagem, 73 (4), e20190031. https://doi.org/10.1590/0034-7167-2019-0031

Conselho federal de enfermagem. Resolução $n^{\circ}$ 358, de 15 de outubro de 2009. Dispõe sobre a Sistematização da Assistência de Enfermagem e a implementação do Processo de Enfermagem em ambientes, públicos ou privados, em que ocorre o cuidado profissional de enfermagem, e dá outras providências. http://www.cofen.gov.br/resoluo-cofen-3582009_4384.html

Daltro, M. R., \& Faria, A. M. (2019) Relato de experiência: Uma narrativa científica na pós-modernidade. Estud. pesqui. psicol., Rio de Janeiro, 19 (1): 223 237. http://pepsic.bvsalud.org/scielo.php?script=sci_arttext\&pid=S1808-42812019000100013

Fernandes, A. M. G., Melo Soares, G. T., Silva Nascimento, L. K. A., Silva Pellense, M. C., Lima Carvalho, G. A. F., \& Sena, D. C. S. (2018). Atuação da enfermagem na detecção precoce e tratamento da sepse na terapia intensiva. Revista humano ser. 3(1): 66-83. https://periodicos.unifacex.com.br/humanoser/article/view/1008

Instituto Latino-Americano Para Estudos da Sepse (Ilas). (2019). Morte por infecção é duas vezes maior na rede pública do que na privada. Brasília: Conselho Federal Medicina. https://ilas.org.br/interacao/?m=201901. 
Research, Society and Development, v. 10, n. 2, e11310212327, 2021

(CC BY 4.0) | ISSN 2525-3409 | DOI: http://dx.doi.org/10.33448/rsd-v10i2.12327

Lobo, S. M. Rezende, E. Mendes, C. L.; Oliveira, M. C. (2019). Mortalidade por sepse no Brasil em um cenário real: projeto UTIs Brasileiras. Revista Brasileira de Terapia Intensiva, 31(1): 1-4. https://doi.org/10.5935/0103-507x.20190008

Maissiat, G. S. Lautert, L. Pai, D. D. \& Tavares, J. P. (2015). Contexto de trabalho, prazer e sofrimento na atenção básica em saúde. Rev. Gaúcha de Enfermagem, 36(2): 42-49. http://dx.doi.org/10.1590/1983-1447.2015.02.51128

Oliveira, C. M., Silva, A. P. F. Silva, M. A. M. (2018) Cuidados de enfermagem ao familiar e à paciente hospitalizada à luz da teoria de Joyce Travelbee. Essentia: Revista de Cultura, Ciência e Tecnologia da UVA. 19(1): 12-21 https://doi.org/10.36977/ercct.v19i1.155

Pereira, A. S., Shitsuka, D. M., Pereira, F. J. \& Shitsuka, R. (2018). Metodologia da Pesquisa Científica. UAB/NTE/UFSM. https://www.ufsm.br/app/uploads/sites/358/2019/02/Metodologia-da-Pesquisa-Cientifica_final.pdf.

Porto, A. O. Leal, C. B. M. Ferreira, R. B. S. F. (2018). Processo de enfermagem aplicado ao paciente com infecção de sítio cirúrgico pós prostatectomia: relato de experiência. Rev. Prev. Infec. e Saúde. 4:7215. https://revistas.ufpi.br/index.php/nupcis/article/view/7215/pdf

Rosa, R. S. Silva, O. C. Picanço, C. M., Biondo, C. S., Andrade, D. M. B., \& Do Prado, I. F. (2018). Intervenções de enfermagem nas alterações dos parâmetros clínicos cardiorrespiratórios em pacientes com sepse. Revista de Enfermagem da UFSM. 8(2): 399-409. https://doi.org/10.5902/2179769224668

Santana, R. A. N. D. S. Marques, S. C. \& Spolidoro, F. V. (2017). Atuação do enfermeiro no paciente séptico em uma unidade de terapia intensiva. Revista Enfermagem em Evidencia, Bebedouro SP, 1 (1): 33-43. http://unifafibe.com.br/revistasonline/arquivos/enfermageme mevidencia/sumario/47/30012018144919.pdf

Silva, A. P. R. M. \& Souza, H. V. (2018). Sepse: importância da identificação precoce pela enfermagem. Revista Pró-UniverSUS. 9(1): 97-100. http://editora.universidadedevassouras.edu.br/index.php/RPU/article/view/1266

Silva, L. S. S. Cordeiro, E. L., Silva, T. D. A., Souza, M. C., Correia, N. D. S., Tenório, M. D. C. S. \& Cavalcanti, S. M. S. (2017). Cuidados de enfermagem a partir das diretrizes internacionais da sepse: uma revisão integrativa. Revista de trabalhos acadêmicos-universo recife, 3(3). http://www.revista.universo.edu.br/index.php?journal=1UNICARECIFE2\&page=article\&op=viewArticle\&path\%5B\%5D=2744.

Veras, R. E. S., Moreira, D. P., Silva, V. D., Rodrigues, S. E. (2019). Avaliação de um protocolo clínico por enfermeiros no tratamento da sepse. Journal of Health and Biological Sciences, 7(3): 292-297. http://dx.doi.org/10.12662/2317-3076jhbs.v7i3.2466.p292-297.2019

Viana, R. A. P. P. Machado, F. R. \& Souza, J. L. A. (2020). SEPSE: Um Problema De Saúde Pública - A atuação e colaboração da Enfermagem na rápida identificação e tratamento da doença. 66p. https://www.ilas.org.br/assets/arquivos/ferramentas/livro-sepse-um-problema-de-saude-publica-coren-ilas.pdf

Waldow, V. R. (2009). Momento de cuidar: momento de reflexão na ação. Revista Brasileira de Enfermagem, 62(1), 140-145. https://doi.org/10.1590/S003471672009000100022

Westphal, G. A. Lino, A. S. (2015). Rastreamento sistemático é uma base do diagnóstico precoce de sepse grave e choque séptico. Revista Brasileira de Terapia Intensiva, 27 (2), 96-101. https://doi.org/10.5935/0103-507X.20150018 